\title{
Formal MES Modeling Framework -Integration of Different Views
}

\author{
Maria Witsch*. Birgit Vogel-Heuser*. \\ *Technische Universität München, Mechanical Engineering Department, \\ Automation and Information System, 85748 Garching bei München, \\ Germany (Tel: 0049-89-289164-40/-01; \\ e-mail:m.witsch@ais.mw.tum.de,vogel-heuser@ais.mw..tum.de).
}

\begin{abstract}
The specification of Manufacturing Execution Systems (MES) as software systems related to the technical process represents a challenge regarding interdisciplinary communication. In this paper, a modeling framework that integrates the different important views for MES specification by coupling their established modeling notations is presented. The main contribution of this framework is the integration of a technical system model, a production process model and an MES functional model with their interconnections and dependencies by a formal link model.
\end{abstract}

\section{MANUFACTURING EXECUTION SYSTEMS}

For a competitive production process, a vertical integration of the automation layer and the Enterprise Resource Planning (ERP) layer is necessary. In addition, the profitability of manufacturers is more and more defined by the economy of their processes. Manufacturing Execution Systems (MES) realize the integration of automation and management ITsystems. While automation usually operates in seconds or milliseconds, Enterprise Resource Planning systems act in middle or long-term range. In order to connect these two extremes regarding their time-horizon, redefinition of data from ERP systems, like orders and production plans or executable data, like work instructions and control parameters, are necessary on the one hand. On the other hand, the mass of data collection during the production execution has to be consolidated to quality data, for example states of order and output figures. (Kletti (2007))

Automated data transfer between automation and ERP by MES can avoid data errors during manual input, accelerate reporting, replace repeating manual operations and by this improve business processes. Additionally, MES improve the visibility of information.

Currently available Manufacturing Execution Systems cover very different ranges of typical functions. For the efficient and successful implementation of MES, an unambiguous specification, exactly defining all functional and technical requirements is therefore highly important. These specifications must be easily understandable by all participating stakeholders within the implementation process. Like shown in (Ricken, Vogel-Heuser (2009, 2010), NAMUR (2009)), no graphical modeling notation supports this specification process adequately at the moment. By developing a graphical modeling notation and integrating different views on the production and business processes, the challenge of interdisciplinary communication can be mastered.

After presenting current problems in the specification process and their consequences for MES-projects, we present a modeling framework for the early phases of the MES engineering process and the integration of different disciplines in this modeling process. In section 4 we formalize the links between these different views, discuss their semantics and finally illustrate benefits on the basis of an example.

\section{CURRENT PROBLEMS IN MES-ENGINEERING PROCESSES}

The information necessary for the specification of MES are spread among multiple co-workers coming from different disciplines with a different focus and working background. Employees responsible for the production process have a different view on the technical and the business process as operators or management personnel. They also know different details about the processes and have diverse requirements concerning the functionality of MES. Moreover, they use different terms and models for describing them. Due to this, it is difficult to integrate all required stakeholders in the specification process.

For MES customers, these difficulties lead to a suboptimal cooperation and islands of knowledge among responsible coworkers and by this to an insufficient use of information potentially available.

Presently, specifications are mostly textual without graphical models. The documents are ambiguous and often not intuitively understandable by people from other disciplines than the author's. Therefore these documents are often not suited for interdisciplinary discussion. For MES customers, this entails missing transparency regarding the concrete functional requirements and selection of MES functions. Additionally, the required transparency for the integration of a new MES into an existing IT-infrastructure can hardly be achieved without an appropriate graphical model.

MES engineering should be integrated into the plant engineering process. By this, comprehensive data can be consistently used not only for MES implementation purposes, but also across different engineering activities. Currently, this data has to be refined with high effort.

Currently, MES vendors often have to detail the specification given by the customer after the order acceptance. This 
detailing process is usually not part of the contract and therefore not paid for by the customer.

The MES vendor also has to identify all necessary data points needed for the new MES system. Important data, which is already recorded by the Process Control System (PCS) system or provided by the machines themselves and integrated into the MES database by import, has to be determined, specified, and interpreted. This interpretation is often difficult, because data points are often neither labeled, described nor self-explanatory, and there are different requirements concerning the level of detail of information, e.g. key performance indicators like equipment effectiveness. The management wants to gather data concerning the effectiveness of a plant as a whole, while the operator wants to get to know detailed information for all machines or modules. Thus, key data is needed in different detail levels for different stakeholders. Existing standards for calculating key data have to be interpreted for specific customer processes. Therefore, MES vendors need a good understanding about the basic production process and all technical aspects of the customer's plant.

\section{MES MODELING APPROACH}

Engineering of MES is an interdisciplinary process where the MES vendor's consultants and IT-specialists as well as the customer's managers, operators, plant engineers and ITspecialists have to work together to integrate their domainspecific requirements and develop a comprehensive solution. For this interdisciplinary process, a simple communication method, easily understandable for all participants, is needed. Intuitively understandable graphic modeling notations fulfill this requirement.

Most of the problems presented before, are getting worse with higher complexity of the plant, but could be solved or even handled more easily with a transparent graphical model of the plant and the planned system.

However, no modeling notation explicitly designed for the modeling of MES was developed so far. Today MES specifications are mainly textual. Some MES vendors developed their own tool-specific models, where each element represents one module of their proprietary MES software solution. Others use modeling notations from associated domains for discussing parts of the requirements, e.g. requirements concerning the integration of MES into the business process. However, these modeling notations are usually limited to the business process level and do not consider automation and technical requirements. (Ricken, Vogel-Heuser (2009, 2010), NAMUR (2009))

The modeling concept presented in the following closes this gap by integrating the necessary views for interdisciplinary MES engineering. It provides three views, each addressing people with different expertise about the modeled system. The first view describes the technical system with its different components in a hierarchical order. The second view specifies the production process. The last model specifies the MES functional view, describing properties and processes a MES should implement.

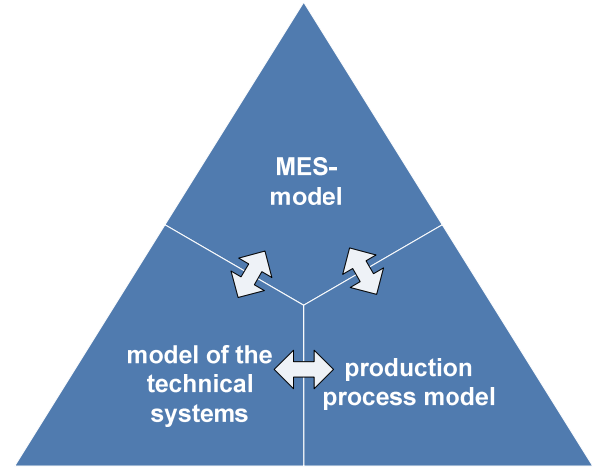

Fig.1. Models of the MES specification approach

A MES Specification Model is defined as a tuple $S_{M E S}=$ $\left\langle M_{M E S}, M_{P P}, M_{T S}, M_{L N}\right\rangle$ constituting one MES Functional Model $\left|M_{M E S}\right|=1$, one Production Process Model $\left|M_{P P}\right|=$ 1, a Technical System Model $\left|M_{T S}\right|=1$ and the MES linking Model $\left|M_{L N}\right|=1$.

The three models and the linkage between them will be described in the following sections. Each model will be introduced with an abstract syntax, which is used later to specify the links between the three models in a formal way.

To distinguish between element connections within one model and connections to elements of different models, the first will be named "connections", the second "links".

\section{MODELS OF THE MES SPECIFICATION}

\subsection{MES Functional Model}

The MES functional model represents the core of this modeling notation. It allows the description of processes realized by the Manufacturing Execution System and displays the functions of MES embedded in interacting IT functionalities and systems.

One of the goals when developing this notation was the usage of state of the art modeling notations. This allows modeling with an established syntax and semantics. The Business Process Model and Notation (BPMN (2010)), developed by the Object Management Group (OMG), appears to be the most powerful modeling notation for business process modeling which is interdisciplinarily understandable. It gains in importance and is supported by an increasing number of software-tools. The previous version (BPMN (2001)) was already considered as the de-facto standard for business process modeling (Decker, Barros (2008)).

The new 2.0 beta version of BPMN defines three types of sub-models within an end-to-end BPMN model: Processes, Choreographies and Collaborations. To model MES functionality and their interaction with surrounding systems, the collaboration model seems to be the most applicable. Due to the extension of elements from version 1.2 (BPMN (2001)) to 2.0 BPMN is becoming a more complex modeling notation. To use the BPMN for MES Functional Model and by this for interdisciplinary MES specification, elements of the BPMN have to be selected and their semantics in the context of MES must be defined. 
A MES Functional Model is a tuple $M_{M E S}=\left\langle D_{M E S}, S D_{M E S}\right\rangle$ with $|D| \geq 1$ a non-empty set of Diagrams $D_{M E S}$ and $|S D| \geq 0$ a set of Subprocess-Diagrams $S D_{M E S}$. To reduce the complexity and to enhance the readability of the definitions we do not write down the index for each element, hence we write $D$ instead of $D_{M E S}$ and so on. The Diagram $D=$ $\langle S L, F O, D O, C O, A F\rangle$ consists of $|S L| \geq 2$ Swimlanes, $|F O| \geq 3$ Flow Objects, $|D O| \geq 0$ Data Objects, $|C O| \geq 2$ Connecting Objects and $|A F| \geq 0$ Artifacts.

The diagram $S D=\langle L, F O, D O, C O, A F\rangle$ constitutes a Subprocess-Diagram with one Lane $l_{m},|F O| \geq 3$ Flow Objects $|D O| \geq 0$ Data Objects, $|C O| \geq 2$ Connecting Objects and $|A F| \geq 0$ Artifacts.

In the following definitions each occurrence of the variable $\boldsymbol{n}$ represents a different, independent variable $\boldsymbol{n} \in \mathbb{N}^{+}$

Swimlanes are defined as a tuple $S L=\langle P, L\rangle$ with Pools $P=\left\{p_{1}, \ldots, p_{n}\right\},|P| \geq 1$ and Lanes $L=\left\{l_{1}, \ldots, l_{n}\right\},|L| \geq 1$. Flow Objects $F O=\langle A, E, G\rangle$ consist of a non-empty set of Activities $A=\left\{a_{1}, \ldots, a_{n}\right\}$ with a set of Events $E=$ $\left\{e_{1}, \ldots, e_{n}\right\},|E| \geq 2$, and a set of Gateways $G=\left\{g_{1}, \ldots, g_{n}\right\}$.

Data Objects are defined as a tuple $D O=\langle S D O, M D O, D S\rangle$ with $S D O=\left\{s d o_{1}, \ldots, s d o_{n}\right\}$ as a set of Single Data Objects, $M D O=\left\{m d o_{1}, \ldots, m d o_{n}\right\}$ as a set of Multi Data Objects and $D S=\left\{d s_{1}, \ldots, d s_{n}\right\}$ as a set of Data Stores. The tuple $C O=\langle S F, D F, M F\rangle$ defines Connecting Objects which consist of a set of Sequence Flows $S F=\left\{s f_{1}, \ldots, s f_{n}\right\}$, a set of Data Flows $D F=\left\{d f_{1}, \ldots, d f_{n}\right\}$ and a set of Message Flows $M F=\left\{m f_{1}, \ldots, m f_{n}\right\}$. Artifacts are defined as a tuple $A F=\langle T A, G R\rangle$ that consists of Text Annotations $T A=$ $\left\{t a_{1}, \ldots, t a_{n}\right\}$ and Groups $G R=\left\{g r_{1}, \ldots, g r_{n}\right\}$.

For the definition of the linkages between the models, elements of Flow Objects are most important and will therefore be presented in more detail below.

For MES specification, a distinction between automatic and manual activities is helpful. Therefore Activities are defined as a tuple $A=\left\langle A_{U S}, A_{M E S}, A_{M}, A_{U}, A_{P P}\right\rangle$ and can be divided in unspecified Activities $A_{U S}=\left\{a_{1}, \ldots, a_{n}\right\}$, automatic Activities (MES Activities) $A_{M E S}=\left\{a_{M E S 1}, \ldots, a_{M E S n}\right\}$, user-software-interactions (User Tasks) $A_{U}=\left\{a_{U 1}, \ldots, a_{U n}\right\}, \quad$ non-software supported Activities (Manual Tasks) $A_{M}=\left\{a_{M 1}, \ldots, a_{M n}\right\}$ and actions performed by the production process (Production Process Activities) $A_{P P}=\left\{a_{P P 1}, \ldots, a_{P P n}\right\}$.

An MES Activity is defined as a generic term for a functionality that shall be performed by the MES in the process. A User Task is a typical "workflow" task where a human actor performs the task with the assistance of the MES software application. A Manual Task is an unmanaged task in the sense that the MES does not track the start and completion of such a task. Actions of the production process which are correctly mapped in the Production Process Model, but have a huge impact on the MES process, can be modeled as Production Process Activity in the MES Functional Model. Events are defined as a tuple $E=\left\langle E_{S}, E_{S T}, E_{L S}, E_{I I}, E_{I T I}, E_{I E I}, E_{I N I}, E_{I T N I}, E_{E}, E_{E E} E_{L E}\right\rangle$ with $E_{S}=\left\{e_{S 1}, \ldots, e_{S n}\right\}$ as a set of Start Events, $E_{S T}=$ $\left\{e_{S T 1}, \ldots, e_{S T n}\right\}$ as a set of Start Timer Events, $E_{L S}=$ $\left\{e_{L S 1}, \ldots, e_{L S n}\right\}$ as a set of Start Link Events, $E_{I I}=$ $\left\{e_{I I 1}, \ldots, e_{I I n}\right\}$ as a set of Intermediate Events, $E_{I T I}=$
$\left\{e_{I T I 1}, \ldots, e_{I T I n}\right\}$ as a set of Intermediate Timer Events, $E_{I E I}=\left\{e_{I E I 1}, \ldots, e_{I E I n}\right\}$ as a set of Intermediate Interrupting Exception Events, $E_{I N I}=\left\{e_{I N I 1}, \ldots, e_{I N I n}\right\}$ as a set of Intermediate Non-interrupting Events, $E_{I T N I}=\left\{e_{I T N I 1}, \ldots, e_{I T N I n}\right\}$ as a set of Intermediate Noninterrupting Timer Events, $E_{E}=\left\{e_{E 1}, \ldots, e_{E n}\right\}$ as a set of End Events, $E_{E E}=\left\{e_{E E 1}, \ldots, e_{E E n}\right\}$ as a set of Exception End Events, $E_{L E}=\left\{e_{L E 1}, \ldots, e_{L E n}\right\}$ as a set of Link End Events.

Gateways are defined as a tuple $G=\left\langle G_{I}, G_{E}, G_{P}\right\rangle$ with $G_{I}=\left\{g_{I 1}, \ldots, g_{I n}\right\}$ as a set of Inclusive Gateways, $G_{E}=$ $\left\{g_{E 1}, \ldots, g_{E n}\right\}$ as a set of Exclusive Gateways and $G_{P}=$ $\left\{g_{P 1}, \ldots, g_{P n}\right\}$ as a set of Parallel Gateways.

\subsection{Production Process Model}

MES activities described in the MES Functional Model affect activities in the production process and vice versa. Therefore the knowledge about the production process should be explicitly modeled. This knowledge can be displayed in dynamic models. Models like UML Activity Diagrams (Rumbaugh, et al. (2005)) or Flowcharts (ISO 10628. (1997)) are a common notation for modeling the production process, but other dynamic modeling notations can also be chosen.

Even though the BPMN's scope is widespread, it is an intuitively understandable language and was therefore chosen for the modeling of production processes. In case of a varying preference by the modeler, the BPMN and its individual parts can be replaced by a less complex language. Links between omitted elements will become irrelevant through this.

A Production Process Model is defined as a tuple $M_{P P}=$ $\left\langle D_{P P}, S D_{P P}\right\rangle$. For $D_{P P}$ and $S D_{P P}$ the same definitions, with all their sub elements, as shown in the last section, for the MES Functional Model are valid. To distinguish between elements from $M_{M E S}$ and $M_{P P}$ we use the indices $M E S$ and $P P$ for each element in section 5 .

\subsection{Technical System Model}

The Technical System Model is used to describe the components of the production plant in a comprehensive way. The basis for the MES-specification is a good understanding of the existing technical system or the part to be optimized. The technical system provides the data, which can be used for MES functions. To use this information about the technical system in interdisciplinary discussions, a simple static model of the technical system is sufficient. This model displays the technological structure of the plant as a whole down to atomic function units as the most detailed level. For the definition of interfaces between MES and technical system, more details about existing data, data quality and semantics of data are helpful in this phase of the engineering process. Therefore, each functional unit can be detailed by attributes concerning its quality and semantics.

A Technical System Model is a tuple $M_{T S}=\langle p, A R, U, S N, U D L\rangle$ with $p$ as a Plant, $A R$ as a nonempty set of Plant Areas $A R=\left\{a r_{1}, \ldots, a r_{n}\right\}, U$ as a nonempty set of Units $U=\left\{u_{1}, \ldots, u_{n}\right\}$ and $S N$ as a non-empty set of Signals $S N=\left\{s n_{1}, \ldots, s n_{n}\right\}$ as well as $U D L$ as a set of User-Defined Layers $U D L=\left\{u d l_{1}, \ldots, u d l_{n}\right\}$. These sets are 
hierarchically structured under the plant, which represents the root-node.

\section{MES LINKING MODEL}

Regarding these three types of models and their kinds of information we define three types of links between the models to support the modeler:

- Data transfer

- Equivalence

- Deployment.

It should be possible to model if there is or should be a real (implemented or to be implemented by software) interface between two elements of different models $\left(M_{M E S}, M_{P P}, M_{T S}\right)$ so that a real dependability between two functions/actions is modeled. This kind of interaction is, concerning its meaning, equivalent to message flows inside the MES and the Production Process Model without a graphical representation. This link shows a real coupling of systems and by this the MES's impact on the production process and the other way round. These links are 1:1 relations.

During the modeling and specification process of MES, there are several situations where process steps of the real production and business process have to be modeled without knowing if it is solely a production step, if it interacts with the MES on a more detailed level or if it could be divided into steps executed by the MES and the plant. At least if the MES is not implemented into an existing plant, but is projected in the early engineering phases of a plant on a less detailed level regarding the description of MES and production process, there are process steps, whose executing system (automation system or MES) part is not specified. These process steps are of interest in both the MES and the production process model. In this case, through the equivalent linkage, it is possible to show that the production process-activity in the MES model and the MES-activity in the production Process model represent the same process step in reality.

The third link guides the modeler and any user of the specification through the different models and helps to understand the plant's entire extent, its production process and the part of the MES in-between. It displays which production process take place on which part of the plant.

For the definition of the MES Linking Model, some additional definitions have to be made.

$\mathrm{O}$ represents the set of objects, including every object from the different views that can be linked to objects from other models $O=\left\{O_{M E S} \cup O_{P P} \cup O_{T S}\right\}$ and the view of an object $o \in O$ is given by the function

$$
\operatorname{view}(o)=\left\{\begin{array}{c}
M E S, \text { if } o \in O_{M E S} \\
T P, \text { if } o \in O_{T P} \\
T S, \text { if } o \in O_{T S}
\end{array} .\right.
$$

The elements that can be start or end of a link are defined as

$$
\begin{gathered}
O_{M E S}=\left\{P_{M E S}, L_{M E S}, A_{M E S}, E_{M E S}, G R_{M E S}, D_{M E S}\right\}, \\
O_{P P}=\left\{P_{P P}, L_{P P}, A_{P P}, E_{P P}, G R_{P P}, D_{P P}\right\} \text { and } \\
O_{T S}=\{A R, U, S N, U D L\} .
\end{gathered}
$$

In general, Diagrams, Gateways, Connection Objects, Link Events and Text Annotations from the MES Functional Model as well as from the Production Process Model cannot be linked to elements in other models.

The Linking Model is a set of Links between the models which are defined as:

$$
L_{L N}=\{(a, b) \in O \times O \mid \operatorname{view}(a) \neq \operatorname{view}(b)\} .
$$

It connects two objects from different views. It is possible to link every object from a view to an object from another view. Links between certain object types are not allowed. For this reason we subsequently define how well-formed links look like and discuss the semantics of links between the views and special object types.

The type of a link is given by $t \in T=\{$ DataTransfer, Equivalence,Deployment $\}$. This type leads to restrictions of the linkable objects:

$$
\begin{gathered}
t=\text { DataTransfer } \\
\rightarrow\left(\forall(a, b) \in L_{L N} \mid a, b \in O_{M E S} \cup O_{P P} \cup O_{T S}\right) \\
t=\text { Equivalence } \rightarrow\left(\forall(a, b) \in L_{L N} \mid a, b \in O_{M E S} \cup O_{P P}\right) \\
t=\text { Deployment } \rightarrow\left(\forall(a, b) \in L_{L N} \mid a \in O_{P P}, \mathrm{~b} \in O_{T S}\right)
\end{gathered}
$$

If two elements are linked by an Equivalence relation, the two elements must have the same name attribute.

Following this definition, it is only possible to link one element to another one. By this we define that for linking a number of elements, they have to be grouped beforehand, and be linked to each other through connecting these groups.

In the following, we discuss the links between the different models:

$$
\begin{array}{ll}
\text { - } & M_{M E S} \text { and } M_{P P}, \\
\text { - } & M_{M E S} \text { and } M_{T S}, \\
\text { - } & M_{P P} \text { and } M_{T S} .
\end{array}
$$

\subsection{Links between MES Functional Model and Technical System Model}

Directed links between $M_{M E S}$ and $M_{T S}$ are defined as $\left(\forall(a, b) \in L_{L N} \mid \begin{array}{c}a \in A_{M E S} \cup E_{M E S} \wedge \mathrm{b} \in S N \cup U D L \wedge \\ t=\text { DataTransfer }\end{array}\right)$ so they have to start from Activities, Events, Pools, Lanes or DataStores and end in Signals or User Defined Layers.

Directed links from TS to MES Objects are defined as $\left(\begin{array}{c|c}\forall(a, b) \in L_{L N} & \begin{array}{c}\mathrm{a} \in S N \cup U D L \wedge \\ b \in A_{M E S} \cup E_{M E S} \cup \mathrm{DS} \cup \operatorname{SL} \wedge \\ t=\text { DataTransfer }\end{array}\end{array}\right)$ and have to start from Signals or User Defined Layers and end in Activities or Events. An Exception here would be the TimeEvent, because it never has a data input.

\subsection{Links between Production Process Model and Technical System Model}

Links between the production process and the technical system can be defined as 


$$
\left(\forall(a, b) \in L_{L N}\left(\begin{array}{c}
(t=\text { DataTransfer } \rightarrow \mathrm{a} \in S N \cup U D L \wedge \\
b \in A_{P P} \cup E_{P P} \cup \mathrm{DS}_{\mathrm{PP}} \cup \mathrm{SL}_{\mathrm{PP}} \vee \\
\mathrm{a} \in A_{P P} \cup E_{P P} \cup \mathrm{DS}_{\mathrm{PP}} \cup \mathrm{SL}_{\mathrm{PP}} \\
\wedge \mathrm{b} \in S N \cup U D L) \vee \\
(t=\text { Deployment } \rightarrow \\
a \in A_{P P} \cup E_{P P} \cup \mathrm{SL}_{\mathrm{PP}} \cup \mathrm{GR}_{\mathrm{PP}} \wedge \\
\left.\mathrm{b} \in \mathrm{O}_{\mathrm{TS}}\right)
\end{array}\right) .\right.
$$

\subsection{Links between MES Functional Model and Production Process Model}

There are a lot of possibilities for Links between $M_{M E S}$ and $M_{P P}:\left(\forall(a, b) \in L \mid a, b \in O_{M E S} \cup O_{P P}\right)$. They can be DataTransfer Links or Equivalence Links. Usually, a message flow coming from the production process initiates an event in the MES model and vice versa. But also other linkages are possible. Table 1 shows all possible Links between $M_{M E S}$ and $M_{P P}$ considering their direction. Impossible linkages are filled grey.

Exemplarily, the top-left cell in table 1 containing $<\mathrm{E}>$ defines that a Pool in a MES Model can have an Equivalence relation to a Pool in the Production Process Model and a Pool in a Production Process Model can have an Equivalence relation to a Pool in the MES Model. In the same cell $<\mathrm{D}>$ defines that a Pool in a MES Model can have a Datatransfer relation to a Pool in the Production Process Model and a Pool in a Production Process Model can have a Datatransfer relation to a Pool in the MES Model. The same links are possible for Lanes to Lanes and Lanes to Pools.

Each activity-type can have an Equivalence-relation to the same activity-type in the other diagram. Manual activities never have a Datatransfer to the other system because they represent an activity that is executed by an operator or user initiated by the system but without any system interaction. An Unspecified Activity can have an Equivalence relation to nearly all activities instead of $A_{M E S}$ for links from $M_{P P} \rightarrow$ $M_{M E S}$ and instead of $A_{P P}$ for links from $M_{M E S} \rightarrow M_{P P} . A_{U S}$ as start for a Equivalence relation between $M_{M E S} \rightarrow M_{P P}$ with $A_{P P}$ as end of the link, $A_{U S}$ has to be an $A_{P P}$.

Events happen in a system or process and are assigned to it. They can initiate another event in the other system by a Datatransfer but not be equivalent to another event. Timer Events are initiated by their given time attribute not by incoming data and cannot have a data input but can be the source for a datatransfer.

From the two models only Equivalence relations can be established between all three kinds of DataObjects.. There can never be a Datatransfer between DataObjects because single and multiple DateObjects are themselves subjects of a Datatransfer and if a datatransfer starts or ends in a Dataobject, this Dataobject should be assigned to the same model as its source or target. Datastores will never send without any activities or events as source or target.

Table 1. Possible Links between $M_{M E S}$ and $M_{P P}$

\begin{tabular}{|c|c|c|c|c|c|c|c|c|c|c|c|c|c|c|c|c|c|c|c|c|c|}
\hline \multirow{2}{*}{\multicolumn{2}{|c|}{$M_{\text {MP }}$}} & \multirow[b]{2}{*}{$\mathbf{P}$} & \multirow[b]{2}{*}{ L } & \multirow[b]{2}{*}{ GR } & \multicolumn{5}{|c|}{ Activity } & \multicolumn{9}{|c|}{ Event } & \multicolumn{3}{|c|}{ DataObject } \\
\hline & & & & & Aus & $A_{\text {MES }}$ & $A_{M}$ & $A_{U}$ & App & $E_{s}$ & $\mathrm{EST}_{\mathrm{ST}}$ & $E_{\|}$ & $E_{T 1} \mid$ & $E_{|E|}$ & $\mathrm{E}_{\mathbb{N 1} \mathbf{I}}$ & $E_{T T N 1}$ & $E_{E}$ & $\mathrm{E}_{\mathrm{EE}}$ & SDO & MDO & DS \\
\hline & & $\begin{array}{l}\langle\mathrm{E}\rangle \\
\langle\mathrm{D}\rangle\end{array}$ & \begin{tabular}{|l|}
$<\mathrm{E}>$ \\
\\
$\langle\mathrm{D}\rangle$ \\
\end{tabular} & $<E>$ & $|\mathrm{D}\rangle$ & $\langle\mathrm{D}\rangle$ & & & $\mid<E>$ & $D>$ & & $D>$ & & & $D>$ & & $<D>$ & $\mid\langle D\rangle$ & & & $|<D\rangle$ \\
\hline & L & $\begin{array}{l}\langle E\rangle \\
\langle D\rangle \\
\end{array}$ & $\begin{array}{l}\langle\mathrm{E}\rangle \\
\langle\mathrm{D}\rangle\end{array}$ & $<E>$ & $|\mathrm{D}\rangle$ & $\langle\mathrm{D}\rangle$ & & & $\mid<E>$ & $D>$ & & D> & & & $D>$ & & $<D>$ & $\mid\langle D\rangle$ & & & $|<D\rangle$ \\
\hline & GR & $\begin{array}{l}\langle\mathrm{E}> \\
\langle\mathrm{D}>\end{array}$ & 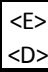 & $\begin{array}{l}\langle E\rangle \\
\langle D\rangle\end{array}$ & $\begin{array}{l}\langle\mathrm{E}\rangle \\
\langle\mathrm{D}\rangle\end{array}$ & $\langle\mathrm{D}\rangle$ & $|<E>|$ & & $\mid \mathrm{E}>$ & $<D$ & $<D$ & $\langle\mathrm{D}\rangle$ & $<D$ & $<D$ & $\mid\langle D\rangle$ & $<\mathrm{D}$ & $|\mathrm{D}\rangle$ & $\mid\langle D\rangle$ & & & $D>$ \\
\hline \multirow{5}{*}{ : } & Aus & $\langle\mathrm{D}\rangle$ & $\mid\langle D\rangle$ & $\langle$ D $\rangle$ & \begin{tabular}{|l}
$\langle E \mathrm{E}\rangle$ \\
$\langle\mathrm{D}\rangle$
\end{tabular} & $\langle\mathrm{D}\rangle$ & $|<E>|$ & $\mid<E>$ & $\mid<E>$ & $\langle\mathrm{D}\rangle$ & $<\mathrm{D}$ & $\langle\mathrm{D}\rangle$ & $<D$ & $<D$ & $\langle D\rangle$ & $<D$ & $<D$ & $<D$ & & & $D>$ \\
\hline & $A_{\text {MES }}$ & $\langle E\rangle$ & $<E>$ & $<E>$ & & $\langle E\rangle$ & & & & & & & & & & & & & & & \\
\hline & $A_{M}$ & & & & $\mid<E>$ & & $\langle\mathrm{E}\rangle$ & & & & & & & & & & & & & & \\
\hline & $\mathrm{Au}$ & & & $\langle E>$ & <E> & $|\mathrm{D}\rangle$ & $|<E\rangle$ & $\begin{array}{l}<\mathrm{D}> \\
<\mathrm{E}>\end{array}$ & $\mid<E>$ & $\langle\mathrm{D}\rangle$ & $<D$ & $\langle\mathrm{D}\rangle$ & $<D$ & $\langle\mathrm{D}\rangle$ & $\mid\langle D\rangle$ & $<\mathrm{D}$ & $|\mathrm{D}\rangle$ & $\mid\langle D\rangle$ & & & $D>$ \\
\hline & App & $\langle\mathrm{D}\rangle$ & $\langle D\rangle$ & $\langle\mathrm{D}\rangle$ & $\langle D\rangle$ & $\langle\mathrm{D}\rangle$ & & & $<E>$ & $\langle\mathrm{D}\rangle$ & $<D$ & $\langle$ D $\rangle$ & $<D$ & $\langle\mathrm{D}\rangle$ & $\langle D\rangle$ & $<D$ & $<D>$ & $|\mathrm{D}\rangle$ & & & $\langle D\rangle$ \\
\hline \multirow{9}{*}{ 荎 } & Es & $<D$ & $<\mathrm{D}$ & $D>$ & $|\mathrm{D}\rangle$ & $<D$ & $<D$ & & & $\langle$ D $\rangle$ & $<\mathrm{D}$ & $<D$ & $<\mathrm{D}$ & & $D>$ & $<D$ & & & & & $D>$ \\
\hline & $E_{S T}$ & D> & $D>$ & $D>$ & $D>$ & D> & & & & $D>$ & & D> & & & $D>$ & & & & & & D> \\
\hline & $E_{\| 1}$ & D> & D> & $\langle\mathrm{D}\rangle$ & $|\mathrm{D}\rangle$ & D> & & & & & & $\langle$ D $\rangle$ & $<D$ & $\langle\mathrm{D}\rangle$ & $\langle D\rangle$ & $<D$ & $|\mathrm{D}\rangle$ & $\mid<D>$ & & & D> \\
\hline & $E_{m 1}$ & D> & $D>$ & $D>$ & D> & $D>$ & & & & $D>$ & & $D>$ & & & D> & & & & & & $D>$ \\
\hline & $E_{|E|}$ & D> & $D>$ & D> & $D>$ & & & & & & & $D>$ & & $\langle\mathrm{D}\rangle$ & $D>$ & & $<D>$ & $\langle\mathrm{D}\rangle$ & & & $D>$ \\
\hline & $E_{|N|}$ & $\langle D\rangle$ & $\langle\mathrm{D}\rangle$ & $\langle\mathrm{D}\rangle$ & $\langle D\rangle$ & $D>$ & & & & $\langle\mathrm{D}\rangle$ & $<\mathrm{D}$ & $\langle D\rangle$ & $<D$ & $<D$ & $\langle\mathrm{D}\rangle$ & $<D$ & $<D$ & $<D$ & & & $D>$ \\
\hline & EITNI & D> & D> & $D>$ & D> & D> & & & & D> & & D> & & & $D>$ & & & & & & D> \\
\hline & $\mathrm{E}_{\mathrm{E}}$ & $\langle\mathrm{D}\rangle$ & $|\mathrm{D}\rangle$ & D> & D> & $\langle\mathrm{D}\rangle$ & & $|\mathrm{D}\rangle$ & & & & $D>$ & & $D>$ & $D>$ & & $\langle\mathrm{D}\rangle$ & $|\mathrm{D}\rangle$ & & & D> \\
\hline & $\mathrm{EEE}_{\mathrm{EE}}$ & $\langle\mathrm{D}\rangle$ & $\mid\langle D\rangle$ & D> & D> & $\langle\mathrm{D}\rangle$ & & & & & & $D>$ & & $D>$ & $D>$ & & $\langle\mathrm{D}\rangle$ & $|\mathrm{D}\rangle$ & & & $D>$ \\
\hline \multirow{3}{*}{ 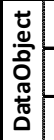 } & SDO & & & & & & & & & & & & & & & & & & $<E>$ & & \\
\hline & MDO & & & & & & & & & & & & & & & & & & & $<E>$ & \\
\hline & DS & $\langle\mathrm{D}\rangle$ & $\mid\langle D\rangle$ & $<D$ & $<D$ & $\langle\mathrm{D}\rangle$ & & $<D$ & & $<D$ & $<D$ & $<D$ & $<D$ & $<D$ & $<D$ & $<D$ & $<D$ & $<D$ & & & $\langle\mathrm{E}\rangle$ \\
\hline
\end{tabular}

Direction: ( $>$ ) Link from MPp to $M_{M E S ;}(<)$ Link from MMEs to $M_{P P} ;(<>)$ Link in both directions possible Type of Linkage: (D) Datatransfer; (E) Equivalence 


\section{MODELING EXAMPLE}

An example for a link between the technical system and the MES Functional Model is the link between $S N$ and $E_{M E S}$. It specifies which signals (Emergency Stop in Fig. 2.) shall be used by the MES and how the technical system and the MES interact directly. By the implementation of the developed
MES it is possible, that these modeled links are not realized as direct interface between the machine and the MES but with an OPC interface for example. However the link shows which signals from the machine are necessary for the requested MES-function.
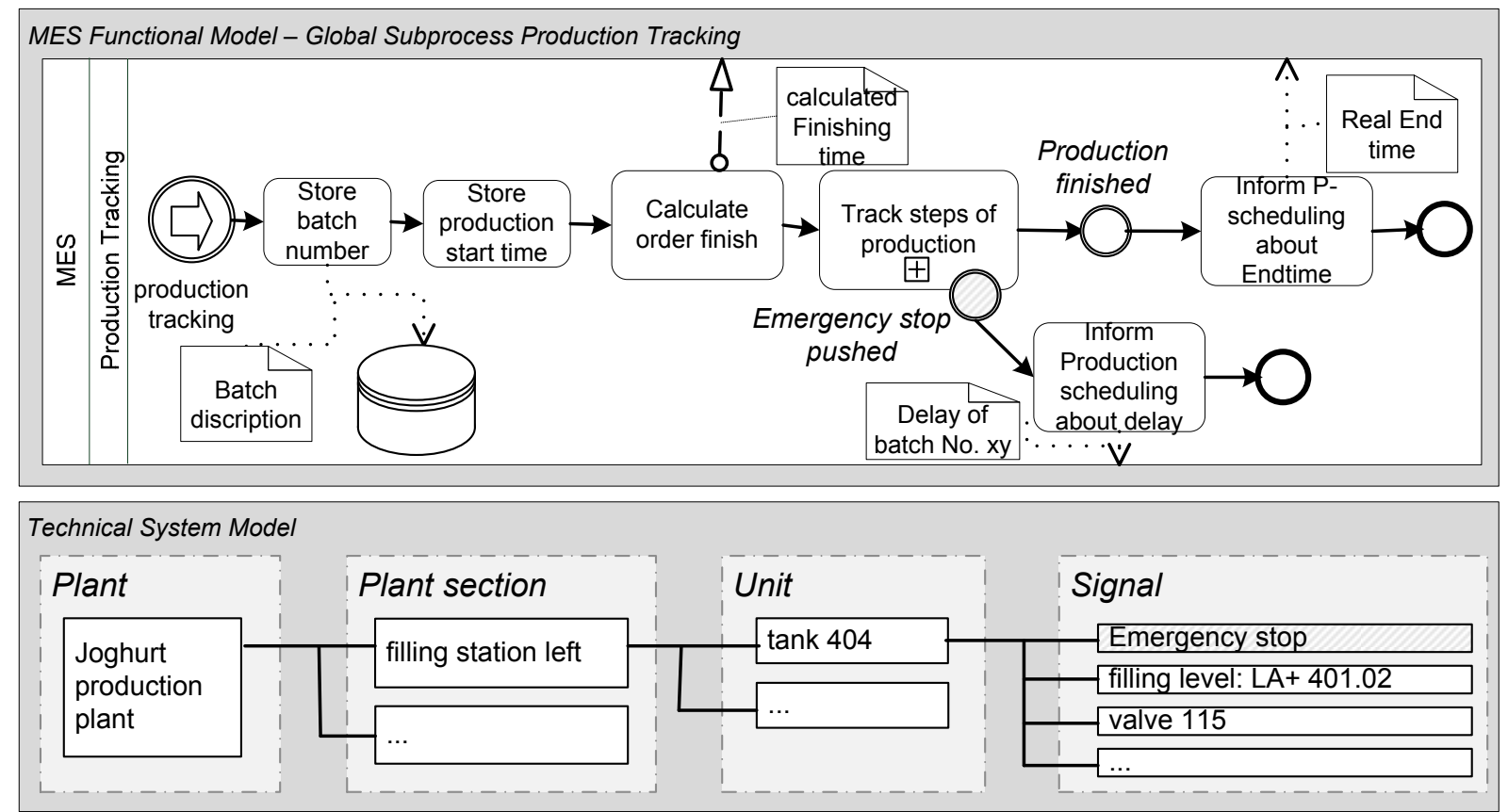

Fig. 2. Modeling Example Excerpt of a MES Specification Model for Link

\section{ACKNOWLEDGEMENTS}

This work is supported by the German Ministry of Education and Research (BMBF) under grant number 01IS09026C.

\section{REFERENCES}

Instrumentation, Systems and Automation Society. (2000) SP95 - Enterprise-Control System Integration.

Object Management Group. (2010). Business Process Model and Notation (BPMN). http://www.omg.org/spec/BPMN/2.0/Beta1/PDF/.

Ricken, M., Vogel-Heuser, B. (2010) Modeling of Manufacturing Execution Systems: an Interdisciplinary Challenge. In: Proc. of ETFA 2010 - 15th IEEE International Conference on Emerging Technologies and Factory Automation. Bilbao, Spain.

Ricken, M., Vogel-Heuser, B. (2009) Engineering von Manufacturing Execution Systems. SPS/IPC Drives Kongress.

Rumbaugh, J., Jacobson, I., Booch, G. (2005) The Unified Modeling Language Reference Manual. Pearson.

ISO 10628. (1997) Flow Diagrams For Process http://www.iso.org/iso/catalogue detail.htm?csnumber= 18721.

Kletti, J. (2007) Manufacturing Execution System $M E S$. Springer. Berlin, Heidelberg, New-York.

Object Management Group. (2001). Business Process Modeling Notation Specification, http://www.omg.org/spec/BPMN/1.2/PDF/.

NAMUR, Worksheet 128 (NA 128) MES Specification and Design based on the Fictitious Example of a Beverage Production Process, 2009.

Decker, G., Barros, A. (2008). Interaction Modeling Using BPMN. In: Business Process Management Workshop, LNCS 4928. (ter Hofstede, A., Benatallah, B., Paik, H.-Y., (Ed.). 208-219. Springer. Berlin, Heidelberg.

Lewis, R. (2001). Modeling control systems using IEC 61499 - Applying function blocks to distributed systems, IEE Control Engineering Series 59, Institution of Electrical Engineers.

Girault, C., Rudiger, V. (2003). Petri Nets for Systems Engineering - A Guide to Modeling, Verification, and Applications, Springer, New York. 